\section{$\underset{\substack{\text { hommes } \\ \text { \& migrations }}}{ }$}

\section{Hommes \& migrations}

Revue française de référence sur les dynamiques

migratoires

$1324 \mid 2019$

Religion et discrimination

\title{
Religion et discrimination
}

\section{Patrick Simon et Liza Rives}

\section{CpenEdition \\ Journals}

\section{Édition électronique}

URL : https://journals.openedition.org/hommesmigrations/8161

DOI : 10.4000/hommesmigrations.8161

ISSN : 2262-3353

\section{Éditeur}

Musée national de l'histoire de l'immigration

\section{Édition imprimée}

Date de publication : 1 janvier 2019

Pagination : 8-9

ISBN : 978-2-919040-44-5

ISSN : $1142-852 X$

\section{Référence électronique}

Patrick Simon et Liza Rives, «Religion et discrimination », Hommes \& migrations [En ligne], 1324 | 2019, mis en ligne le 01 janvier 2019, consulté le 07 janvier 2022. URL : http://journals.openedition.org/ hommesmigrations/8161; DOI : https://doi.org/10.4000/hommesmigrations.8161 


\section{Religion \\ et discrimination}

\section{Patrick Simon,}

directeur de recherche à l'Institut national d'études démographiques (Ined) et chercheur associé au Centre d'études européennes de Sciences Po

\section{Liza Rives,}

chargée de mission à l'Alliance de recherche sur les discriminations (Ardis).

$\underline{\mathbf{D}}$ ans ses déclinaisons institutionnelles, spirituelles ou sociales, la religion - et singulièrement l'islam - est au cœur des débats de société dans de nombreux pays européens et outre-Atlantique. La crispation à l'égard de l'islam en France se lit dans de nombreux faits divers, dans les déclarations de certains commentateurs politiques et essayistes, et dans les sondages d'opinion. Le dernier sondage sur «les fractures françaises» réalisé en juillet 2018 par Ipsos pour la Fondation Jean Jaurès, Sciences Po et le journal Le Monde montre que, si $92 \%$ des personnes interrogées pensent que la religion catholique est compatible avec les valeurs de la société française et $81 \%$ pensent de même pour la religion juive, elles ne sont plus que $42 \%$ à le penser pour l'islam. Les enquêtés sont également $73 \%$ à trouver que la religion musulmane cherche à imposer son mode de fonctionnement aux autres alors qu'ils ne sont respectivement que $22 \%$ et $21 \%$ à le penser pour le catholicisme et le judaïsme. Ces perceptions relativement négatives de l'islam sont bien entendu influencées par le débat sur la laïcité, et la vague récente d'attentats qui a renforcé l'amalgame entre musulmans et terroristes, et plus généralement la conviction que l'intégrisme et la radicalisation à fondement religieux est propre à l'islam (47\% des personnes interrogées considèrent que l'islam «porte malgré tout en lui des germes de violence»).
Ces doutes ou hostilité à l'égard de la place de l'islam dans la société française se déclinent dans de nombreux domaines de la vie sociale où les discriminations fondées sur la religion tendent à prendre de l'ampleur. Ce sont ainsi plus de $40 \%$ des personnes en île-de-France se déclarant musulmanes qui rapportent en 2015 avoir fait l'expérience d'une discrimination religieuse au cours des cinq dernières années, alors qu'elles n'étaient que $5 \%$ à le faire en 2008 ${ }^{1}$. Plusieurs testings ont identifié une pénalité spécifique contre les musulman.e.s dans la sélection des candidatures à des offres d'emploi². Ces faits convergents montrent qu'il existe un lien spécifique entre religion et discrimination.

Face à ces discriminations, la politique de lutte contre les discriminations est en difficultés. Cette proposition peut sembler paradoxale puisque la religion bénéficie d'une protection constitutionnelle rappelée dans l'article 1 de la Constitution de 1958 «[La République] assure l'égalité de tous les citoyens devant la loi sans distinction d'origine, de race ou de religion » - et qu'elle figure dans la liste des motifs discriminatoires dans la loi française depuis 2001. De

1. Mireille Eberhard, Patrick Simon, «Perceptions et expériences des discriminations en Île-de-France ", Ardis-CGET-DDD, 2015

2. Voir notamment Marie-Anne Valfort, Discriminations religieuses à l'embauche: une réalité, Paris, Institut Montaigne, 2015. 
fait, les libertés religieuses protégées dans les textes fondamentaux sont également prises en compte dans la loi de séparation de l'Église et de l'État et ses prolongements dans ce qu'on peut appeler une politique renouvelée de la laïcité. La norme de non-discrimination est ainsi confrontée à la norme laïque qui en relativise l'application. Il ne s'agit pas seulement d'une question juridique ouverte par la loi de 2004 sur les signes religieux à l'école et les dispositions encadrant l'expression religieuse sur le lieu de travail, mais plus généralement des «accommodements» à réaliser pour aménager la place des religions dans une société fortement sécularisée. Les débats sur la façon dont le dispositif de lutte contre les discriminations est capable d'intervenir contre les conséquences d'une islamophobie de plus en plus décomplexée ne sont pas uniquement juridiques et commencent par la définition du problème. Le concept même d'islamophobie est contesté et il est souvent délicat d'isoler la référence religieuse dans des discriminations de plus en plus intersectionnelles dans la mesure où elles combinent le genre, l'origine, la religion et la couleur de peau. Ainsi, l'objet lui-même des discriminations à raison de la religion échappe largement à l'analyse et au traitement juridique.

C'est pour interroger les relations entre discrimination et religion que l'Alliance de recherche sur les discriminations (Ardis) ${ }^{3}$ a organisé un colloque en octobre 2016 dont les textes composant ce dossier sont pour la plupart issus. Il aborde les liens entre discrimination et religion au travers de différentes disciplines: droit, géographie, sociologie, science politique et psycho-sociologie en Europe et aux États-Unis. Une partie du dossier porte sur la nature et les formes des discriminations faites aux musulmans, et notamment sur le processus de racialisation de la religion qui est à l'œuvre avec l'islam. Revenant sur la définition de l'islamophobie, Nasar Meer identifie sa spécificité à travers la racialisation de la religion et la mobilisation de stéréotypes et de préjugés combinant des références à l'origine ou la race, la religion et la culture de telle sorte que l'islamophobie est devenue un racisme à part entière. À l'échelle de la région parisienne, Kawtar Najib propose une analyse géographique de l'islamophobie tout en mettant l'accent sur l'intersectionnalité du phénomène, à partir des données du Collectif contre l'islamophobie en France (CCIF) ; programme de recherche financé par la Commission européenne (SAMA Project). Elle met en perspective les actes antimusulmans avec d'autres types de discriminations telles que le sexisme, le racisme, l'âgisme et le classisme. Juliette Galonnier revient quant à elle sur l'imbrication du religieux et du racial qui sous-tend la perception des musulmans en France et aux ÉtatsUnis, deux pays ayant eu des histoires contrastées avec le monde musulman et ayant entretenu des rapports distincts, voire concurrents, avec les questions raciales et religieuses. Enfin, Martin Aranguren et Francesco Madrisotti ont conduit une expérimentation originale pour établir l'effet propre du signe religieux, ici un foulard, sur les interactions quotidiennes.

Une autre partie du dossier traite des politiques et des approches juridiques consacrées à la religion et aux discriminations. S'appuyant sur son expérience de consultant, Edwin Hatton montre comment, dans les années 2010, les politiques de lutte contre les discriminations ont progressivement laissé place à une politique de promotion de la laïcité dans les plans de formation et dans les politiques territoriales. Ce changement de paradigme est également perceptible dans l'évolution de l'interprétation du concept de laïcité et de sa réglementation étudiées par Christine Pauti. Elle souligne les effets paradoxaux de ce déplacement du concept de laïcité sur le principe de non-discrimination et son application dans le service public et, plus largement, dans l'espace public. Dans une comparaison entre la France et la Belgique, Léopold Vanbellingen appréhende la polysémie de la notion d'«entreprise neutre». En quoi cette notion vient réinterroger l'application horizontale classique des concepts juridiques de non-discrimination et de liberté de religion dans la sphère du travail, et l'articulation entre les droits individuels du travailleur d'une part, et le droit de l'employeur ou l'intérêt de l'entreprise d'autre part?

Ces approches multidisciplinaires alertent sur la montée des discriminations religieuses et de comparaisons internationales à l'encontre des musulmans et sur les ambiguïtés du cadre laïc face à la reconnaissance de l'islam et aux aménagements sociétaux nécessaires pour réduire les exclusions.

3. L'Alliance de recherche sur les discriminations était un réseau de chercheurs dont l'objectif visait à valoriser et soutenir des recherches en île-de-France menées sur la thématique des discriminations. De 2012 à 2018, cette initiative a reçu le soutien financier de la région île-de-France dans le cadre des dispositifs DIM (Domaine d'intérêt majeur). 